\title{
A Distributed Approach to Constructing Travel Solutions by Exploiting Web Resources
}

\author{
Oudom Kem, Flavien Balbo and Antoine Zimmermann \\ Univ Lyon, MINES Saint-Étienne, CNRS, Laboratoire Hubert Curien, UMR 5516, F-42023, Saint-Étienne, France \\ Email: \{oudom.kem, flavien.balbo, zimmermann\}@emse.fr
}

\begin{abstract}
Many advanced traveler information systems (ATIS) provide travel solutions that are limited, by technical obstructions or by design, in terms of geographical coverage, transport services, and/or travel modes. However, using existing ATIS in an integrated manner can broaden the coverage of travel solutions, while preserving the advantages of each system. This paper presents an approach to exploit web resources such as ATIS, data sources and services to construct travel solutions. More precisely, it focuses on itinerary formulation and discovery of resources relevant to each itinerary. We propose a semantic model for describing and interlinking geographic entities in relation to web resources, creating a graph of related entities. Itinerary formulation and resource discovery are achieved via exploring the graph. To this end, we propose an adapted version of multi-agent $A^{*}$ algorithm.
\end{abstract}

Keywords-Multi-agent A* algorithm; Seed graph; Semantic Web; Travel solution

\section{INTRODUCTION}

Numerous advanced traveler information systems (ATIS) are in operation. However, most ATIS are designed to cover only certain types of travel modes, transport services, and/or geographical areas. For instance, there are systems designed only for private vehicles such as PersianGulf [1], for public public transport (e.g. TCL for public transport in Lyon) and for a specific geographical coverage (e.g. Transport for London for the city of London). Therefore, regardless of the significant number of existing ATIS, travelers are often required to consult multiple ATIS and/or to seek for travelrelated information from various other sources to acquire sufficient information for their trip. When unexpected events such as delays or accidents occur during their trips, travelers need to search for alternatives to adapt to those events.

Pertinent resources for building travel solutions, such as ATIS and other data sources as well as services are available on the Web. Using them complementarily can improve three main aspects of travel solutions. (1) Adaptability: Solutions can be built using resources, which are not statically predefined, but are chosen according to actual requests from travelers. (2) Completeness: The use of multiple existing ATIS as well as other resources in an aggregated manner can widen the coverage of solutions. (3) Personalization: Travelers' preferences and constraints can be taken into account both in the process of discovering resources and in the computation of solutions.
The aim of our research is to design a framework that is able to automatize the entire process of formulating itineraries, searching for relevant web resources, accessing them and integrating acquired information and services to construct travel solutions tailored to each traveler's request. Fig. 1 illustrates the abstract model of the framework, emphasizing the four integral components. Request analysis component extracts essential information such as the origin, the destination and any indicated preferences or criteria from a given request. Discovery component searches for an optimal itinerary between an origin and a destination as well as resources that provide information and/or services assisting travel along an itinerary. Taking an itinerary, its relevant resources, and travel information as well as services acquired previously as an input, solution construction component's roles comprise integrating the acquired heterogeneous data, coordinating services, retrieving travel-related data and services (e.g. weather, gas stations) if necessary, and eventually assigning information and services to relevant segment(s) of the itinerary to build a travel solution. Solution refinement component monitors resources associated with a chosen itinerary for updates and unexpected events (e.g. delays, accidents), and adapts solutions to consider changes.

The focus of this paper is the discovery component. A distributed approach to itinerary formulation and web resource discovery is presented. In this approach, a semantic model for describing and interlinking geographic/administrative entities in function of web resources is proposed. We adapt a multi-agent $A^{*}$ algorithm to perform resource discovery and itinerary formulation.

\section{SEED GRAPH}

An itinerary is composed of one or more segments. Each segment consists of a starting and an ending locations that are geographic entities. Generally, geographic entities can be under a certain hierarchy, namely administrative entities (e.g. a city, a country). We use the notion of seed as an intermediary between geographic/administrative entities and resources relevant to the entities. Each seed describes an entity in terms of resources relevant to that entity and connections to other entities in function of connecting resources.

These connections link each seed to other related seeds, and thus forming a seed graph. Seed data model consists of: 


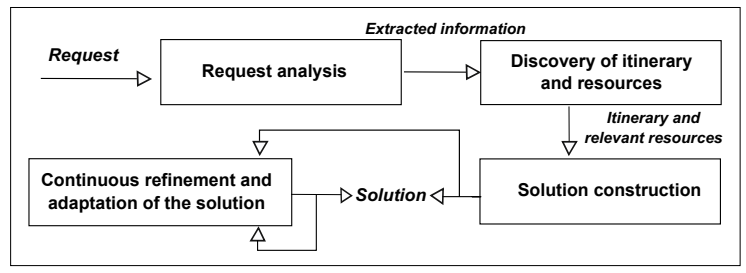

Figure 1. Abstract model of the framework

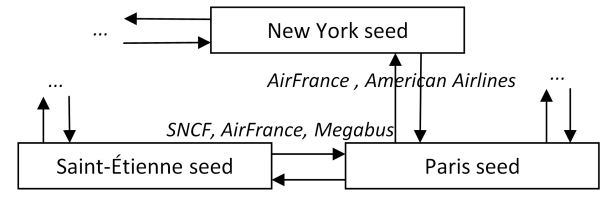

Figure 2. Example of a seed graph

- Topic refers to a geographic or administrative entity with which the seed is associated.

- Associated resources provide information and/or services (e.g. ATIS, hotel websites) that are relevant to a seed's topic. Each associated resource corresponds to a topic, which is determined by information or services that it offers (e.g. transport, weather, tourism).

- Connections between seeds are composed of a target seed and one or multiple connecting resources. The semantics of a connection is that the entity (i.e. topic) of the target seed is accessible from the entity of the source seed. Information and/or services on how to travel from the source to the target entity can be acquired from the connecting resources.

Fig. 2 illustrates an example of a small portion of a seed graph. Let us examine one seed in the graph. The SaintÉtienne seed is associated with the entity Saint-Étienne (i.e. its topic). From Saint-Étienne, it is possible to go to Paris (i.e. topic of the target seed). SNCF, AirFrance and Megabus (i.e. connecting resources) provide information on how to travel from Saint-Étienne to Paris.

To have a fully distributed approach, seed graph is also distributed on the Web. Seeds are described using RDF (Resource Description Framework) [2]. The use of IRI (Internationalized Resource Identifier) in RDF to reference resources in a uniform and web-compatible manner provides a convenient and proper way to describe connections to associated resources and to other seeds.

To model seeds, an ontology, entitled Seed ontology, was created. As depicted in Fig. 3, the ontology consists of two classes and four properties, and reuses a property, primaryTopic, of FOAF vocabulary [3].

In practice, it would require a lot of resources and efforts to collect information about available resources and create seeds for all the geographic and administrative entities. Therefore, ideally, from people involved in the domain (e.g. authority of a city, transport operators) to individuals willing

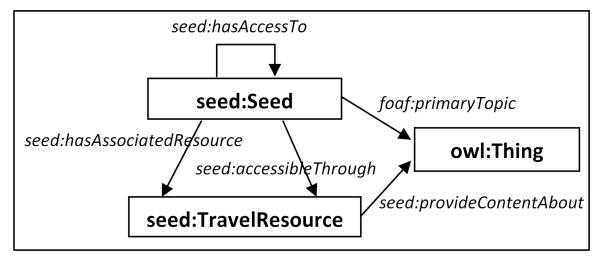

Figure 3. Diagram of the Seed ontology

to contribute, anyone is able to create seeds for a set of geographic and/or administrative entities of their interest, link them to existing seeds, and make them available on the Web. In the same manner as how the Web has evolved so far, this would allow the seed graph to scale and grow.

\section{ALGORITHM FOR ITINERARY FORMULATION AND RESOURCE DISCOVERY}

\section{A. Problem description}

Given a seed graph, itinerary formulation and resource discovery are realized by exploring the graph. This exploration can be formulated as a search problem as follows:

- States: A set of states $S$ corresponds to seeds in a graph.

- Initial state: An initial state $s_{i} \in S$ corresponds to the seed whose topic is the origin of a requested itinerary.

- Actions: Actions are connecting resources of connections among seeds. In each state, there is a set of applicable actions, referring to connecting resources of connections to other seeds that can be found in a seed.

- Transition model: Executing an action $a \in$ Actions in a state $s \in S$ leads to a resulting state $s^{\prime}$ which is the target seed of a connection. In our problem, actions and their cost are initially unknown, and are to be discovered during the search.

- Goal test: A state $s$ is a goal state if its topic is the destination of a requested itinerary.

- Path cost: Path cost is the actual travel cost such as travel distance, duration, and monetary cost.

Solution consists of a path from an initial state to a goal state (i.e. itinerary) and a sequence of actions (i.e. connecting resources providing instructions on how to travel along the itinerary) leading to the goal state. Referring to the graph in Fig. 2, if the request is to travel from Saint-Étienne to New York, the problem is to find a path between Saint-Étienne and New York and its connecting resources. Let us suppose that the best solution is:

$$
\text { Saint-Étienne } \stackrel{S N C F}{\longrightarrow} \text { Paris } \stackrel{\text { AirFrance }}{\longrightarrow} \text { New York }
$$

The itinerary is to travel from Saint-Étienne to Paris, and then to New York. Being web-based ATIS, SNCF and AirFrance provide information on how to travel from SaintÉtienne to Paris and from Paris to New York, respectively. 


\section{B. The discovery algorithm}

The proposed algorithm is based on the classic $A^{*}$ algorithm and multi-agent approach. Some basic parts concerning the usage of agents (e.g. exchanging messages) are inspired, with adaptations to our problem, from a generic multi-agent version of $\mathrm{A}^{*}, \mathrm{MA}-\mathrm{A}^{*}$ [4]. Multi-agent systems have been shown to be suitable for solving problems in an open and complex environment where required resources are distributed [5]. They are applicable in our context because seed graph and resources are distributed over a highly dynamic and open environment, the Web. There are some distributed and parallel variants of $\mathrm{A}^{*}$ such as [4] and [6]. While many works address the issues of distributed and parallel settings such as separating search spaces and distributing knowledge, none, to our knowledge, address the problem where actions and their cost are initially unknown and are to be discovered during the search. In addition, in our problem, acquiring an action cost is a time-consuming task that also needs to be considered.

Agents involved in the algorithm are search agents (SA) and resource agents (RA). SAs execute the discovery algorithm. They communicate with RAs to request for costs to travel between seeds. RAs act as an intermediary between the framework and resources. An RA is responsible for a resource, and it knows how to access the resource. RAs accept queries from SAs. They access resources to obtain requested information and/or services and return them to SAs. Another role of RAs is to monitor changes in processed resources (i.e. currently being used by travelers). In this respect, changes can be taken into account to adapt travel solutions for travelers (in solution refinement component).

In the algorithm (Algorithms 1-3), each agent maintains an open-list (OL) of candidate states for expansion, a closedlist (CL) of expanded states, an action-list (AL) containing actions available for execution, and a pending-list (PL) storing actions whose costs are being requested. AL is used to divide workloads among agents, and is synchronized to prevent different agents from executing the same action.

An execution cycle of an agent $A$ begins by processing received messages (A:1 L:3). Then, it selects a state $s$ which has the lowest cost from its OL to expand (A:1 L:5). If $s$ is a goal state, $A$ initiates goal verification procedure (A:2 $\mathrm{L}: 2-5)$. Otherwise, $s$ is expanded. In that case, an expansionmessage about $s$ is broadcasted. Receiving agents process the message and react accordingly (A:3 L:15-22). If $s$ with a better cost is already in their OL, they suggest it to $A$ via a suggest-state-message. In this way, $A$ can expand $s$ with the best cost currently known among agents. When expanding $s$, if $A$ discovers actions, it broadcasts a new-actions-message containing the actions so that other agents help execute the actions. After the expansion, $A$ selects an action $a$ at the top of its AL to execute (A:1 L:6). In AL, actions are organized in first-in, first-out fashion since action costs are unknown.
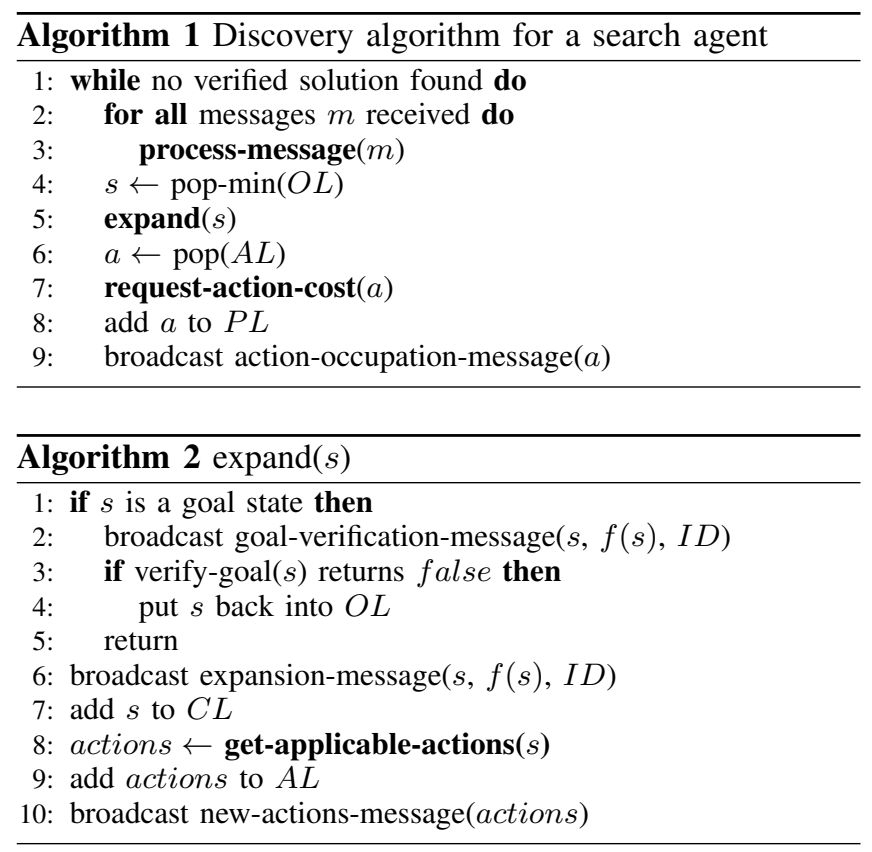

An action-occupation-message about $a$ is broadcasted, and receiving agents update their AL accordingly. Executing $a$ in $s$ will lead to a successor $s^{\prime}$. To calculate the cost of $s^{\prime}, f\left(s^{\prime}\right)$, it is necessary to acquire the cost of $a, c\left(s, a, s^{\prime}\right)$. A sends a non-blocking request for $c\left(s, a, s^{\prime}\right)$ to an RA associated with $a$. Once it receives the response in the form of responseaction-cost message, it computes $f\left(s^{\prime}\right) . s^{\prime}$ is added to OL if it is not in OL and CL. If $s^{\prime}$ is in the OL, but its cost is higher than the new $s^{\prime}$, it is replaced by the new $s^{\prime}$. In case $s^{\prime}$ has already been expanded (i.e. in CL) and the new $s^{\prime}$ has a lower cost, $s^{\prime}$ is reopened. Otherwise, it is discarded.

1) Goal verification procedure: When a goal state is expanded, the expanding agent broadcasts a goal-verificationmessage to verify the optimality of the solution. A solution with a goal state $s^{*}$ is optimal if there exists no state $s$ in the entire system where $f(s)<f\left(s^{*}\right)$. To verify that property, assuming all messages in the queue are processed, each agent checks its OL, AL and PL. If there is any candidate state $s$ in OL where $f(s)<f\left(s^{*}\right)$, the solution is not verified. If there are actions in AL or PL, the solution is not verified. The cost of the actions in these two lists are still unknown, so it is impossible to determine the cost of their resulting states. If the solution is not verified, $s^{*}$ is reinserted into OL. In this way, only states having lower cost than $s^{*}$ will be expanded. If a better goal state is discovered, it replaces the existing goal state in OL. When a solution is verified, the expanding agent traces back for the path and executed actions. Then, it broadcasts a terminating message.

2) Concurrent action selection: If multiple agents have taken the same action, the first agent, determined by the time-stamp included in the action-occupation-message, that chose the action can execute the action. If the time-stamps 


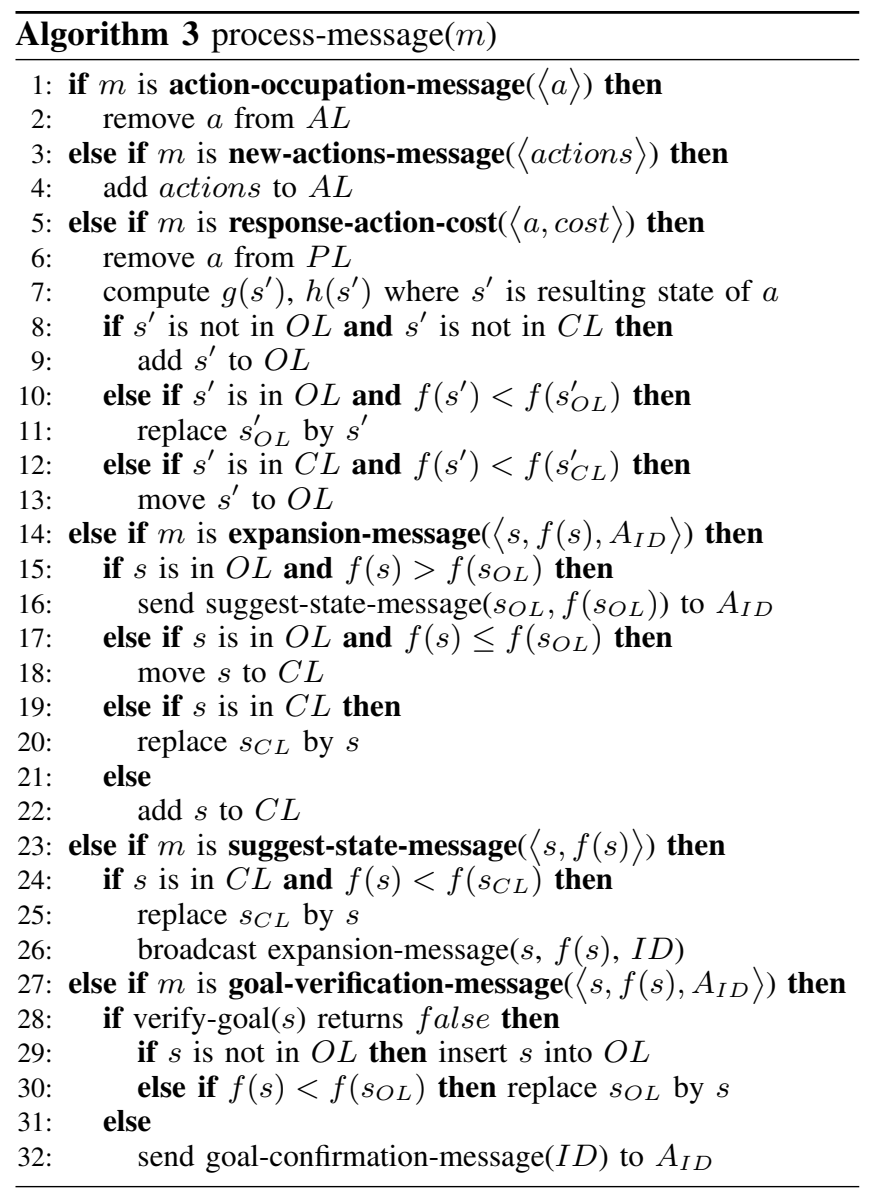

are identical, the agent with smaller ID continues the execution. Since these heuristics are known by all agents, they can determine whether to continue or stop.

3) Concurrent expansion of the same state: If agents detect a concurrent expansion of the same state $s$, they proceed in the following way. If $s$ has different costs, the agent expanding $s$ with the lowest cost continues. If $s$ has the same cost, the agent that has the lowest number of states in its OL continues (this information is included in the messages concerning concurrent expansion). If the conflict is still not resolved, the agent with the smallest ID continues.

4) Termination: The execution of the algorithm is terminated in two situations. First, a verified solution is found. Second, the entire state space has been explored. We reach the end of a state space when all agents have no states in OL, no actions in AL and no pending requests in PL.

5) Optimality: The algorithm terminates by finding a path to a goal state with an optimal cost if one exists, assuming the following properties: (1) The heuristic function $h$ is consistent. (2) The search space is finite. (3) All sent messages arrive at their destinations. (4) For every request for an action cost, we get a response. (5) All operations take a finite amount of time. To prove the optimality of our algorithm, we employ the proof by contradiction by assuming the contrary. We consider 3 following cases as also addressed in the generic MA-A* [4]. (1) The algorithm terminates at a non-goal state. This case is impossible because the goal verification procedure only starts when expanding a goal state. (2) The algorithm terminates at a non-optimal goal state. In the goal verification procedure, a goal state $s^{*}$ is verified only when, for every agent, it has the lowest cost in the OL, and there exists no action in the AL or PL that may lead to a state $s$ where $f(s)<f\left(s^{*}\right)$. Therefore, when a non-optimal goal is being verified, there must be a state $s^{\prime}$ belonging to the optimal path, in the OL or as a resulting state of an action in the AL or PL, where $f\left(s^{\prime}\right)<f\left(s^{*}\right)$. In such case, $s^{*}$ is not verified. (3) The algorithm does not terminate. First, if an optimal solution exists, the algorithm terminates after the solution is verified. Second, when all states in the search space are expanded, the algorithm terminates. Third, the search space is finite, so the number of time a state $s$ can be reopened from the $\mathrm{CL}$ is also finite.

\section{CONCLUSiON}

The proposed approach allows independent resources to be described and interlinked such that they can be discovered. The distributed and open architecture enables flexible entries and exits of resources. Moreover, employing multi-agent approach facilitates the use of distributed and heterogeneous resources and the personalization process. The feasibility of the approach depends on people's will to describe and interlink seeds by adhering to a common model, but a worldwide adoption of a specification is not uncommon on the Internet. However, the number of web resources are immense. The scalability of the approach needs to be ensured and will be addressed in our future work.

\section{REFERENCES}

[1] M. Khanjary, K. Faez, M. Meybodi, and M. Sabaei, "Persiangulf: An autonomous combined traffic signal controller and route guidance system," in VTC Fall, Sept 2011, pp. 1-6.

[2] R. Cyganiak, D. Wood, and M. Lanthaler, "RDF 1.1 Concepts and Abstract Syntax, W3C Recommendation 25 February 2014," Feb. 25 2014. [Online]. Available: http://www.w3.org/TR/2014/REC-rdf11-concepts-20140225/

[3] D. Brickley and L. Miller, "FOAF Vocabulary Specification 0.99, Namespace Document 14 January 2014 Paddington Edition," Jan. 14 2014. [Online]. Available: http://xmlns.com/foaf/spec/

[4] R. Nissim and R. I. Brafman, "Multi-agent A* for parallel and distributed systems," in Proceedings of the 11th International Conference on Autonomous Agents and Multiagent SystemsVolume 3. International Foundation for Autonomous Agents and Multiagent Systems, 2012, pp. 1265-1266.

[5] P. Bogg, G. Beydoun, and G. Low, Intelligent Agents and Multi-Agent Systems: PRIMA 2008, Hanoi, Vietnam, December 15-16, 2008. Proceedings. Springer Berlin Heidelberg, 2008, ch. When to Use a Multi-Agent System?, pp. 98-108.

[6] A. Kishimoto, A. S. Fukunaga, and A. Botea, "Scalable, parallel best-first search for optimal sequential planning," in ICAPS, 2009. 